\title{
Uso do Protocolo de Manchester em pacientes pediátricos: limitações descritas na produção científica
}

\author{
Manchester Protocol use in pediatric patients: limitations described in scientific production \\ Uso del Protocolo de Manchester en pacientes pediátricos: limitaciones descritas en la \\ producción científica
}

Maicon Douglas Xavier Braga ${ }^{1 *}$,Dayane Barbosa Pereira ${ }^{1}$, Samiria Junia Reis Coelho ${ }^{1}$, Eliete dias de Araújo ${ }^{1}$, Vilma Silva Lima², Joany Alves Damasceno ${ }^{3}$.

\begin{abstract}
RESUMO
Objetivo: O objetivo desse trabalho foi verificar, por meio de uma revisão integrativa, como é descrita a sensibilidade e a especificidade do uso do Sistema de Triagem de Manchester (STM) na população pediátrica. Métodos: Trata-se de uma revisão integrativa realizada por meio de buscas nas bases de dados Scielo, LILACS e Pubmed. Foram utilizados descritores disponíveis no índice de descritores de ciência de saúde (DECS): triagem, serviços medico de emergência, enfermagem, crianças, pediatria. Resultados: Após a busca e análise dos artigos considerados elegíveis percebeu-se que os resultados indicaram pouca expressividade na produção científica nas bases regionais (LILACS e Scielo); observou-se ainda que a maior parte da amostra de artigos é oriunda da busca no PUBMED. Quanto a metodologia utilizada pelos autores houve predominância do tipo observacional e prospectivo e o número de sujeitos alocados nos estudos variou de 191 a 64.653. De um modo geral, o Sistema de Triagem de Manchester (MTS) não foi considerado com alta especificidade ou sensibilidade no que se refere ao atendimento de urgência na população pediátrica. Considerações finais: O MTS apresentou limitações importantes nos estudos analisados no que se refere ao atendimento e classificação na urgência pediátrica e em condições clínicas associadas a processos infecciosos e outros distúrbios, e maior confiabilidade nas situações de emergência ou que exigem atendimento imediato.
\end{abstract}

Palavras chave: Triagem, Serviço Médico de Emergência, Enfermagem, Criança, Pediatria.

\begin{abstract}
Objective: The purpose of this study was to verify, through an integrative review, how the (Manchester Triage System (MTS) use specificity is described in the pediatric population. Methods: This is an integrative review carried out through searches in the SciELO, LILACS and Pubmed databases. The descriptors available were unrelated to the descriptors of health science (DECS): screening, emergency medical services, nursing, children, pediatrics. Results: After the search and analysis of the data considered eligible, the results were evidenced in the scientific expression in the regional bases (LILACS and SciELO); it is still observed that most of the sample of articles comes from the search in PUBMED. The use of the research method was predominantly observational and prospective, and the number of individuals studied ranged from 191 to 64,653. Overall, the Manchester Screening System (MTS) was not considered to have high specificity or sensitivity with regard to emergency care in the pediatric population. Final considerations: MTS presented important limitations in the studies analyzed in regard to care and classification in pediatric urgency and clinical conditions associated with infectious processes and other disorders, and greater reliability in emergency situations or requiring immediate care.
\end{abstract}

Keywords: Triage, Emergency Medical Service, Nursing, Child, Pediatrics.

\footnotetext{
${ }^{1}$ Enfermeiros graduados pela Faculdade de Saúde Ibituruna (FASI). Montes Claros - MG.

*E-mail: maiconmoc2007@hotmail.com

${ }^{2}$ Graduanda do curso de Psicologia da Faculdade de Saúde Ibituruna (FASI). Montes Claros - MG.

${ }^{3}$ Graduanda do curso de Enfermagem da Faculdade de Saúde Ibituruna (FASI). Montes Claros - MG.
} 


\section{RESUMEN}

Objetivo: El objetivo de este trabajo fue verificar, por medio de una revisión integrativa, como se describe la sensibilidad y la especificidad del uso del sistema de clasificación de manchester (STM) en la población pediátrica. Métodos: Se trata de una revisión integrativa realizada por medio de búsque das en las bases de datosScielo, LILACS y Pubmed. Se utilizaron descriptores disponibles en el índice de descriptores de ciencia de salud (DECS): clasificación, servicios medico de emergencia, enfermería, niños, pediatría. Resultados: Después de la búsqueda y análisis de los artículos considerados elegibles se percibió que los resultados indicaron poca expresividad en la producción científica en las bases regionales (LILACS y Scielo); se observó que la mayor parte de la muestra de artículos procede de la búsque da en PUBMED. Encuanto a la metodología utilizada por los autores hub o predominio del tipo observacional y prospectivo y el número de sujetos a signados en los estudios varía de 191 a 64.653. En general, el Sistema de Clasificación de Manchester (MTS) no fue considerado con alta especificidad o sensibilidad en lo que se refiere a la atención de urgencia en la población pediátrica. Consideraciones finales: EI MTS presentó limitaciones importantes en los estudios analizados en lo que se refiere a la atención y clasificación en la urgencia pediátrica y en condicionesclínicasasociadas a procesosinfecciosos y otrosdisturbios, y mayor confiabilidaden las situaciones de emergencia o que exigen atención inmediata.

Palabras clave: Triage, ServicioMédico de Emergencia, Enfermería, Niño, Pediatría.

\section{INTRODUÇÃO}

Os serviços de emergência tem enfrentado grandes desafios principalmente no que se refere à necessidade de atendimento rápido e humanizado em um contexto de dificuldades de atendimento relacionadas a superlotação dos pronto atendimentos no Brasil. Uma forma de garantir os princípios de resolutividade e integralidade nos serviços de urgência e emergência ou até mesmo no atendimento geral é a utilização de ferramentas de classificação de risco baseadas em fortes evidências científicas para garantir agilidade e eficiência no atendimento (BRASIL, 2013).

Criado na Inglaterra, o sistema de triagem mais usado nos hospitais atualmente é o Sistema de Triagem de Manchester (STM); sua avaliação é baseada em fluxogramas selecionados pelo enfermeiro através da queixa principal e da história clínica do paciente definindo a priorização do atendimento (AMTHAUERC e CUNHA MLC, 2016).

No tocante à população pediátrica, existem grandes dificuldades para os profissionais de saúde no atendimento dessa faixa etária que exige do enfermeiro mais habilidade durante 0 atendimento, uma avaliação clínica mais criteriosa e uma escuta mais qualificada. A observância destas particularidades pode diminuir a chance de uma classificação subestimada reduzindo os riscos relacionados ao agravamento do quadro clínico (AMTHAUER C e CUNHA MLC, 2016).

Como forma de avaliar a confiabilidade e a validade do STM em atendimento pediátrico é necessário conhecer as limitações relacionadas ao uso do protocolo nessa faixa etária considerando se o mesmo é sensível a condições clínicas variadas e se pode, eventualmente, apresentar menor especificidade em situações de risco no atendimento a crianças. Assim, objetivo desse trabalho foi verificar, por meio de uma revisão integrativa, como é descrita a sensibilidade e a especificidade do uso do STM na população pediátrica.

\section{MÉTODOS}

Trata-se de uma revisão integrativa realizada por meio de buscas nas bases de dados Scielo, LILACS e Pubmed. Foram utilizados descritores disponíveis no índice de descritores de ciência de saúde (DECS): triagem, serviços medico de emergência, enfermagem, crianças, pediatria. Os critérios de inclusão utilizados foram artigos produzidos nos 10 anos, devido a carência de estudos na literatura nacional, utilizou dados internacional, disponível na base de dados ou na plataforma do periódico e que respondiam a questão da pesquisa.

Em seguida, procedeu-se a leitura dos títulos e resumos, efetuada a leitura completa dos textos componentes da amostra e a respectiva extração dos dados ou respostas sobre a questão da pesquisa. Os 
dados foram consolidados em forma de um quadro sinóptico (Quadro 2) e realizada a categorização das respostas.

\section{RESULTADOS}

Quadro 1 - Quadro sinóptico com os cruzamentos das palavras chaves disponível nos descritores de ciência da saúde.

\begin{tabular}{|c|c|c|c|c|c|}
\hline Cruzamentos & $\begin{array}{c}\text { Base de } \\
\text { dados }\end{array}$ & Resultados & $\begin{array}{c}\text { Artigos } \\
\text { selecionados }\end{array}$ & $\begin{array}{c}\text { Artigos } \\
\text { repetidos/excluídos }\end{array}$ & $\begin{array}{c}\text { Artigos } \\
\text { elegíveis }\end{array}$ \\
\hline $\begin{array}{c}\text { Manchester (and) } \\
\text { triage system } \\
\text { (and) child } \\
\text { (Best match } \\
\text { searchinformation, } \\
\text { Meshterms: child; } \\
\text { triage) }\end{array}$ & Pubmed & 32 & 09 & 12 & 12 \\
\hline $\begin{array}{c}\text { Manchester (and) } \\
\text { triage system } \\
\text { (and) children }\end{array}$ & Pubmed & 38 & 15 & 01 & 00 \\
\hline $\begin{array}{c}\text { sistema triagem } \\
\text { (and) manchester } \\
\text { (and) crianças }\end{array}$ & Lilacs & 03 & 01 & 00 & 0 \\
\hline $\begin{array}{c}\text { Sistema (and) } \\
\text { triagem (and) } \\
\text { manchester }\end{array}$ & Scielo & 10 & 00 & & 0 \\
\hline Total & --- & --- & 25 & 13 & 12 \\
\hline
\end{tabular}

Fonte: Dados da pesquisa, 2018.

Após a busca e análise dos artigos considerados elegíveis percebeu-se que os resultados indicaram pouca expressividade na produção científica nas bases regionais (LILACS e Scielo); observou-se ainda que a maior parte da amostra de artigos é oriunda da busca na PUBMED.

Quanto a metodologia utilizada pelos autores houve predominância do tipo observacional e prospectivo e o número de sujeitos alocados nos estudos variou de 191 a 64.653. Foram abordados os sujeitos de formas variadas incluindo a verificação das classificações pelos sistemas de triagem nos registros a partir da comparação com métodos diferentes de triagem até a verificação de grupos distintos de pacientes para acompanhamento e classificação.

Quadro 2 - Quadro sinóptico de resultados dos artigos encontrados na base de dados da PubMed.

\begin{tabular}{|l|l|l|l|l|}
\hline Artigo & Autor/ano & Metodologia & N (sujeitos) & Resultado \\
\hline $\begin{array}{l}\text { Validation of different } \\
\text { pediatric triage } \\
\text { systems in the } \\
\text { emergency } \\
\text { department }\end{array}$ & $\begin{array}{l}\text { AEIMCHANBANJON } \\
\text { G, K; PANDEE, U } \\
(2017)\end{array}$ & $\begin{array}{l}\text { Estudoobservacional, } \\
\text { prospectivo, } \\
\text { unicêntrico. }\end{array}$ & $\begin{array}{l}920 \\
\text { pacientespedi } \\
\text { átricos }\end{array}$ & $\begin{array}{l}\text { O MTS ilustrou } \\
\text { sensibilidade } \\
\text { De 57\% e } \\
\text { especificidade } \\
\text { de } 69 \% \text { como } \\
\text { validade em } \\
\text { atendimento } \\
\text { pediátrico. }\end{array}$ \\
\hline $\begin{array}{l}\text { Can urgency } \\
\text { classification of the } \\
\begin{array}{l}\text { Manchester triage } \\
\text { system predict serious }\end{array}\end{array}$ & $\begin{array}{l}\text { NIJMAN R et al., } \\
\text { (2015) }\end{array}$ & $\begin{array}{l}\text { Estudoobservacionalpr } \\
\text { ospectivo }\end{array}$ & 1255 crianças & $\begin{array}{l}\text { O MTS } \\
\text { mostrou } \\
\text { sensibilidade } \\
\text { de previsão }\end{array}$ \\
\hline
\end{tabular}




\begin{tabular}{|c|c|c|c|c|}
\hline $\begin{array}{l}\text { bacterial infections in } \\
\text { febrile children? }\end{array}$ & & & & $\begin{array}{l}\text { para infecções } \\
\text { bacterianas } \\
\text { graves (SBIs) } \\
\text { e pouca } \\
\text { sensibilidade o } \\
\text { modelo que } \\
\text { inclua idade, } \\
\text { sexo e } \\
\text { temperatura. }\end{array}$ \\
\hline $\begin{array}{l}\text { Validity of the } \\
\text { Manchester triage } \\
\text { system in } \\
\text { paediatricsemergency } \\
\text { care }\end{array}$ & $\begin{array}{l}\text { ROUKEMA J et al., } \\
(2015)\end{array}$ & $\begin{array}{l}\text { Estudo } \\
\text { Retrospectivoobservaci } \\
\text { onal }\end{array}$ & $\begin{array}{l}1065 \\
\text { pacientes }\end{array}$ & $\begin{array}{l}\text { O MTS } \\
\text { apresentou } \\
\text { moderada } \\
\text { sensibilidade e } \\
\text { especificidade } \\
\text { no } \\
\text { atendimento } \\
\text { de emergência } \\
\text { pediátrico. }\end{array}$ \\
\hline $\begin{array}{l}\text { Undertriage in the } \\
\text { Manchester triage } \\
\text { system: an } \\
\text { assessment of } \\
\text { severity and options } \\
\text { for improvement }\end{array}$ & $\begin{array}{l}\text { SEIGER N et al., } \\
(2011)\end{array}$ & $\begin{array}{l}\text { Estudo de caso, } \\
\text { observacional } \\
\text { prospectivo }\end{array}$ & $\begin{array}{l}13.408 \\
\text { pacientes }\end{array}$ & $\begin{array}{l}\text { O MTS } \\
\text { apresentou } \\
\text { sensibilidade e } \\
\text { especificidade } \\
\text { moderada em } \\
\text { atendimento } \\
\text { emergência } \\
\text { pediátrica. }\end{array}$ \\
\hline $\begin{array}{l}\text { Accuracy of triage for } \\
\text { children whit chronic } \\
\text { Illness and infectious } \\
\text { symptoms }\end{array}$ & $\begin{array}{l}\text { SEIGER N et al., } \\
(2013)\end{array}$ & $\begin{array}{l}\text { Estudoobservacionale } \\
\text { prospectivo }\end{array}$ & 8592 crianças & $\begin{array}{l}\text { O MTS } \\
\text { apresentou } \\
\text { validade baixa } \\
\text { em crianças } \\
\text { com doenças } \\
\text { crônicas em } \\
\text { relação a } \\
\text { crianças sem } \\
\text { doenças } \\
\text { crônicas }\end{array}$ \\
\hline $\begin{array}{l}\text { Manchester triage } \\
\text { system in paediatrics } \\
\text { emergency care: } \\
\text { prospective } \\
\text { observational study }\end{array}$ & $\begin{array}{l}\text { VEEN MV et al., } \\
(2008)\end{array}$ & $\begin{array}{l}\text { Estudoobservacionalpr } \\
\text { ospectivo }\end{array}$ & $\begin{array}{l}13554 \\
\text { crianças }\end{array}$ & $\begin{array}{l}\text { MTS } \\
\text { apresentou } \\
\text { validade } \\
\text { moderada, há } \\
\text { dificuldades na } \\
\text { classificação } \\
\text { de crianças } \\
\text { menores }\end{array}$ \\
\hline $\begin{array}{l}\text { Safety of the } \\
\text { Manchester Triage } \\
\text { System to Detect } \\
\text { Critically III Children at } \\
\text { The Emergency } \\
\text { Department }\end{array}$ & $\begin{array}{l}\text { ZACHARIASSE JM } \\
\text { et al., (2016) }\end{array}$ & Estudo observacional & $\begin{array}{l}50062 \text { visitas } \\
\text { de crianças }\end{array}$ & $\begin{array}{l}\text { O MTS precisa } \\
\text { de } \\
\text { modificações } \\
\text { para oferecer } \\
\text { segurança na } \\
\text { triagem de } \\
\text { crianças } \\
\text { menores e } \\
\text { com } \\
\text { comorbidades. }\end{array}$ \\
\hline
\end{tabular}




\begin{tabular}{|c|c|c|c|c|}
\hline $\begin{array}{l}\text { Improving the } \\
\text { Manchester Triage } \\
\text { System for Pediatric } \\
\text { Emergency Care: An } \\
\text { International } \\
\text { Multicenter Study }\end{array}$ & $\begin{array}{l}\text { SEIGER N ET } \\
\text { AL.,2014 }\end{array}$ & $\begin{array}{l}\text { Estudo retrospectivo e } \\
\text { prospectivo. }\end{array}$ & $\begin{array}{l}64.653 \\
\text { crianças }\end{array}$ & $\begin{array}{l}\text { O MTS } \\
\text { modificado com } \\
\text { o uso de } \\
\text { discriminadores } \\
\text { e inclusão de } \\
\text { sinais vitais } \\
\text { anormais } \\
\text { apresentou } \\
\text { melhor } \\
\text { performance } \\
\text { que o original. }\end{array}$ \\
\hline $\begin{array}{l}\text { Alarming signs in the } \\
\text { Manchester Triage } \\
\text { System: A Tool to } \\
\text { Identify Febrile } \\
\text { Children at Risk of } \\
\text { Hospitalization }\end{array}$ & $\begin{array}{l}\text { LERLAND Y } \\
(2013)\end{array}$ & $\begin{array}{l}\text { Estudo observacional } \\
\text { Prospectivo }\end{array}$ & $\begin{array}{l}2455 \text { crianças } \\
\text { com menos de } \\
16 \text { anos }\end{array}$ & $\begin{array}{l}\text { O uso de } \\
\text { discriminadores } \\
\text { e sinais de } \\
\text { alarme no MTS } \\
\text { melhora a } \\
\text { classificação na } \\
\text { urgência e } \\
\text { indica o risco } \\
\text { de } \\
\text { hospitalização } \\
\text { de crianças } \\
\text { febris. }\end{array}$ \\
\hline $\begin{array}{l}\text { Safety of the } \\
\text { Manchester Triage } \\
\text { System to identify less } \\
\text { urgent patients in } \\
\text { pediatric emergency } \\
\text { care: a prospective } \\
\text { observational study }\end{array}$ & VEEN MV (2011) & $\begin{array}{l}\text { Estudo observacional } \\
\text { prospectivo }\end{array}$ & 191 pacientes & $\begin{array}{l}\text { O MTS é } \\
\text { menos eficaz } \\
\text { para identificar } \\
\text { o risco de } \\
\text { hospitalização } \\
\text { de crianças } \\
\text { menores de } 1 \\
\text { ano, com } \\
\text { problemas } \\
\text { gastrintestinais } \\
\text { ou febre. }\end{array}$ \\
\hline $\begin{array}{l}\text { The Manchester triage } \\
\text { system: improvements } \\
\text { for pediatric } \\
\text { emergency care }\end{array}$ & VEEN MV (2012) & $\begin{array}{l}\text { Estudo observacional } \\
\text { prospectivo }\end{array}$ & $\begin{array}{l}11481 \\
\text { pacientes }\end{array}$ & $\begin{array}{l}\text { Modificações } \\
\text { no MTS para a } \\
\text { população } \\
\text { pediátrica } \\
\text { produzem } \\
\text { maior } \\
\text { especificidades } \\
\text { para } \\
\text { classificação do } \\
\text { risco de } \\
\text { hospitalização }\end{array}$ \\
\hline $\begin{array}{l}\text { Repeatability of the } \\
\text { Manchester Triage } \\
\text { System for children }\end{array}$ & VEEN MV (2010) & $\begin{array}{l}\text { Estudo observacional } \\
\text { prospectivo }\end{array}$ & 198 pacientes & $\begin{array}{l}\text { O MTS mostrou } \\
\text { segurança de } \\
\text { uso pela } \\
\text { repetibilidade } \\
\text { de resultados } \\
\text { em cenários } \\
\text { simulados e } \\
\text { casos reais. }\end{array}$ \\
\hline
\end{tabular}

Fonte: Dados da pesquisa, 2018. 


\section{DISCUSSÃO}

Em um estudo desenvolvido com o intuito de comparar diferentes sistemas de triagem pediátricos utilizados na Tailândia observou-se que o MTS não apresentou o melhor resultado ficando atrás de outros como Emergency Severity Index (ESI) e o Australasian Triage Scale (ATS) com uma sensibilidade de $57 \%$ e uma especificidade de $69 \%$. Segundo os autores o ESI apresentou-se como o sistema de triagem de melhor aplicabilidade por causa do alto grau de predição à admissão (AEIMCHANBANJONG K e PANDEE U, 2017).

Em se tratando da capacidade de predizer a ocorrência de quadros infecciosos mais sérios como septicemia ou meningite bacteriana o MTS apresenta importantes limitações sendo considerado como um sistema de triagem com baixa habilidade na predição destas situações (mesmo estando presentes no MTS importantes preditores de infecção bacteriana séria), pois utiliza-se de outra forma os preditores de infecção sendo mais voltados para a classificação de urgência (NIJMAN R et al., 2015).

De um modo geral, o MTS não foi considerado com alta especificidade ou sensibilidade no que se refere ao atendimento de urgência na população pediátrica. Nos casos de urgência efetivamente constatada o MTS é pouco sensível, entretanto os casos de emergência são classificados de forma razoavelmente segura em crianças (ROUKEMA J et al., 2015).

Como o MTS utiliza os discriminadores "choque, respiração anormal comprometimento de vias aéreas e paciente irresponsivo", como nem todos os pacientes se encaixam nestes discriminadores percebe-se que as crianças com sinais vitais anormais que não se enquadram nesta descrição podem não ser reconhecidas como muito urgentes para o atendimento(SEIGER $\mathrm{N}$ et al., 2011).

Analisando outras pesquisas desse mesmo autor, modificações foram feitas no MTS para atender as crianças que apresentavam sintomas de doenças infecciosas e foi observado que o sistema apresentou um desempenho melhor em reconhecer o grau de urgência com a inclusão dos sinais vitais anormais (SEIGER $\mathrm{N}$ et al.,2013).

Em outra análise que buscou comparar a classificação de crianças com doenças crônicas e sintomas infecciosos com um grupo sem doenças crônicas percebeu-se que o MTS teve maior dificuldade em classificar adequadamente o primeiro grupo. A dificuldade de classificação de crianças com doenças crônicas pode ser resultado da falta de sensibilidade do MTS para esta condição (SEIGER N et al., 2015).

No que se refere aos testes de validade do MTS comparando com um padrão de referência por urgência, os resultados foram positivos em relação ao fator preditivo em avaliar as urgências, mas apresenta limitações em crianças que apresentam problemas médicos não relacionados a trauma (VEEN MV et al.,2008).

O sistema também apresentou dificuldade em identificar crianças com necessidade de internação em unidades de terapia intensiva, isso se deve ao fato da ausência de comorbidades como discriminador nos fluxogramas delimitando a segurança do MTS (ZACHARIASSE JM et al., 2016).

Como forma de melhorar a especificidade, outros autores sugerem a implementação de sinais de alarme ou gravidade nos discriminadores e na utilização dos fluxogramas o que poderia resultar em mais sensibilidade para identificar crianças febris com risco de hospitalização (LERLAND Y et al., 2013). Outras modificações foram desenvolvidas e implementadas e resultaram em uma validade mais confiável na versão do MTS modificado comparando com versão original, apesar de que não houve modificações na sensibilidade, mas especificidade o que trouxe significativa melhora, mostrando mais capacidade de avaliar pacientes pediátricos em situações de urgência (VEEN MV et al., 2012).

Em pesquisas realizadas para testar a capacidade do MTS na classificação de pacientes menos urgentes, o sistema apresentou desempenho positivo em crianças maiores, mas apresentou resultado negativo em crianças menores de um ano que apresentavam sintomas clínicos com risco de internação (VEEN MV et al., 2011). VeenMV et al (2010) referem ainda que o MTS mostrou maior validade no atendimento da emergência pediátrica que em outras condições menos graves ou que exigissem atendimento imediato. 


\section{CONCLUSÃO}

O MTS apresentou limitações importantes nos estudos analisados no que se refere ao atendimento e classificação na urgência pediátrica e em condições clínicas associadas a processos infecciosos e outros distúrbios, e maior confiabilidade nas situações de emergência ou que exigem atendimento imediato. Neste sentido foi possível notar que seria necessária a inclusão de discriminadores mais específicos para a população pediátrica, em especial para crianças menores, incluindo lactentes e neonatos.Notou-se que a produção científica brasileira é ainda incipiente e pouco expressiva, sugere-se que novos estudos sejam realizados investigando na realidade brasileira a validade do MTS em crianças e que viabilizem adaptações de acordo com as necessidades de cada faixa etária e de casos onde a criança apresente comorbidades e doenças crônicas.

\section{REFERÊNCIAS}

1. AMTHAUER C, CUNHA MLC. Sistema de Triagem de Manchester: principais fluxogramas, discriminadores e desfechos dos atendimentos de uma emergência pediátrica. Rev. Latino-Am. Enfermagem, Ribeirão Preto, 2016. 24(2779): 1-8.

2. AEIMCHANBANJONG K, PANDEE U. Validation of different pediatric triage systems in the emergency department. World J Emerg Med. 2017. 8(3): 223-227.

3. BRASIL. Ministério da Saúde. Secretaria de Atenção à Saúde. Departamento de Atenção Especializada. Manual instrutivo da Rede de Atenção às Urgências e Emergências no Sistema Único de Saúde (SUS). Brasília: Editora do Ministério da Saúde, 2013. 84 p.

4. LERLAND Y et al. Alarming Signs in the Manchester Triage System: A Tool to Identify Febrile Children at Risk of Hospitalization. The Journal of pediatrics. 2012; 1(162): 1-8.

5. NIJMAN R et al. Can Urgency Classification of the Manchester Triage System Predict Serious Bacterial Infections in Febrile Children? Archives of disease in childhood. 2015.8 (96): 715-22..

6. ROUKEMA J et al. "Validity of the Manchester Triage System in paediatric emergency care" Emergency medicine journal : EMJ. 2015. 12(23): 906-10.

7. SEIGER $\mathrm{N}$ et al. Undertriage in the Manchester triage system: an assessment of severity and options for improvement. Archives of Disease in Childhood. 2011. 7(96): 653-657.

8. SEIGER N et al. Accuracy of Triage for Children With Chronic Illness and Infectious Symptoms. Pediatrics. 2013. 6(132): 1-8.

9. SEIGER $\mathrm{N}$ et al. Improving the Manchester Triage System for pediatric emergency care: an international multicenter study. PloS one. 2014. 1(9): 1-8.

10. VEEN MV et al. Manchester triage system in paediatric emergency care: prospective observational study. BMJ. 2008; 1(337): 1-8.

11. VEEN MV. Safety of the Manchester Triage System to identify less urgent patients in paediatric emergence care: a prospective observational study. Archives ofdisease in childhood. 2011; 1(96): 513-8.

12. VEEN MV. The Manchester triage system: Improvements for paediatric emergency care. Emergency medicine journal : EMJ. 1(29): 654-9.

13. VEEN MV. Repeatability of the Manchester Triage System for children. Emergency medicine journal: EMJ. 2010; (1)27: 512-6.

14. ZACHARIASSE JM. et al. Safety of the Manchester Triage System to Detect Critically III Children at the Emergency Department. The Journal of Pediatrics , v. $177,232-237 p$. 